Minnesang IN NeUer Sicht 
Günther Schweikle

\section{Minnesang \\ in neuer Sicht}

Verlag J. B. Metzler

Stuttgart - Weimar 
Die Deutsche Bibliothek - CIP-Einheitsaufnahme

Schweikle, Günther:

Minnesang in neuer Sicht / Günther Schweikle.

- Stuttgart ; Weimar : Metzler, 1994

ISBN 978-3-476-00981-4

\section{ISBN 978-3-476-00981-4 \\ ISBN 978-3-476-03515-8 (eBook) \\ DOI $10.1007 / 978-3-476-03515-8$}

Dieses Werk einschließlich aller seiner Teile ist urheberrechtlich geschützt. Jede Verwertung außerhalb der engen Grenzen des Urheberrechtsgesetzes ist ohne Zustimmung des Verlages unzulässig und strafbar. Das gilt insbesondere für Vervielfältigungen, Übersetzungen, Mikroverfilmungen und die Einspeicherung und Verarbeitung in elektronischen Systemen.

(C) 1994 Springer-Verlag GmbH Deutschland Ursprünglich erschienen bei J. B. Metzlersche Verlagsbuchhandlung und Carl Ernst Poeschel Verlag GmbH in Stuttgart 1994 


\section{INHALT}

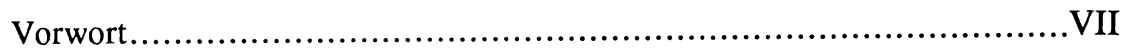

Mittelalterliche Realität in deutscher höfischer Lyrik

und Epik um 1200

Die frouwe der Minnesänger. Zu Realitätsgehalt und Ethos

des Minnesangs im 12. Jh

Der Stauferhof und die mhd. Lyrik, im besonderen zur

Reinmar-Walther-Fehde und zu Hartmanns herre

Humor und Ironie im Minnesang

Zur Edition mittelhochdeutscher Lyrik.

Grundlagen und Perspektiven

Vom Edieren mittelhochdeutscher Lyrik. Theorie und Praxis.

Pseudo-Neidharte?

War Reinmar 'von Hagenau' Hofsänger zu Wien?

Textkritik und Interpretation. Heinrich von Morungen

'Sit siu herzeliebe heizent minne' (MF 132,19) ................................ 216

Eine Morungen-Parodie Walthers? Zu MF 145,33 .......................... 265

Doppelfassungen bei Heinrich von Morungen ............................. 278

Minne und Mâze. Zu 'Aller werdekeit ein füegerinne'

(Walther 46,32)

Steckt im Sumerlaten-Lied Walthers von der Vogelweide

(L 72,31) ein Gedicht Reinmars des Alten?

Die Fehde zwischen Walther von der Vogelweide und Reinmar

dem Alten. Ein Beispiel germanistischer Legendenbildung

Neidhart: Nu ist vil gar zergangen (Hpt. 29,27). Zur Geschichte

eines Sommerliedes (SL 26)

Dörper oder Bauer. Zum lyrischen Personal im Werk Neidharts

Nachweise

Weitere Arbeiten zum Minnesang 


\section{VORWORT}

Mit dieser Sammlung lege ich eine Reihe früherer Aufsätze zum Minnesang vor - weniger als persönliche Bilanz, eher als ein Rückblick auf ein Stück Forschungsgeschichte: den Übergang von der älteren Minnesang-Philologie zu neueren Versuchen, die Minnesangdeutungen wieder stärker auf die mittelalterlichen Handschriften und deren Auswertung auszurichten. Ihre seinerzeit mißtrauisch oder gar ablehnend registrierten Thesen ordnen sich neuerdings meist in einen allgemeinen Konsens ein. Da die einzelnen Arbeiten z.T. recht abgelegen veröffentlicht wurden, mag eine zusammenhängende Publikation nicht gänzlich überflüssig sein.

Aufsätze sind oft wie Versuchsballone, Testfahrten zur Erprobung, wie der Wind weht, oder konkret, wie weit eine These trägt. Zumindest bei mir war dies so, wie schon mein Mitstreiter in Sachen Minnesang, Helmut Tervooren, einmal bemerkte (ZfdA 99, S. 171). Es war vor 30 Jahren nicht ganz einfach, gegen althergebrachte wohletablierte Forschungsmeinungen anzuschreiben, zumal in der (damals) ungesicherten Position eines Assistenten. So geriet ich denn auch mit meinem ersten Versuch (Minne und Mâze, 1963) in gewisse Turbulenzen, denen dann allerdings ein befreiender und beflügelnder Durchbruch folgte, als Hugo KUHN das Skript für die DVjS annahm und zum Weitermachen ermunterte, ebenso wie Friedrich OHLY, der einen weiteren Versuch (Textkritik und Interpretation, 1964) in der ZfdA publizierte. In den auf diese ersten Versuche folgenden Arbeiten habe ich dann auch weiterhin v.a. nicht genügend abgesicherte Forschungsmeinungen im Visier gehabt, besonders diejenigen, die auf den Postulaten der germanistischen Koryphäen von Karl LachmanN, Moritz HaUpt bis hin zu Carl von Kraus fußten (und fußen).

Mein methodischer Ausgangspunkt war stets die in den Handschriften faßbare Dokumentation der Texte. Die möglichst unvoreingenommenen 
Untersuchungen ihres Wortlauts in ihren einzelnen Fassungen und der Art und Weise ihrer Stellung im handschriftlichen Kontext führten nicht selten zu anderen als den vormaligen Ergebnissen der Forschung. Sie betrafen die (heute weitgehend akzeptierte) Deutung der Funktionen des Minnesangs innerhalb der mittelalterlichen historischen Realität (um den unglücklichen Ausdruck 'Sitz im Leben' zu vermeiden), seiner 'poetischen Machart' als fiktionale Spielweit, als weitausgreifende Metapher für die Lebensproblematik des höfischen Publikums und seiner Sänger (Mittelalterliche Realität ... , 1982). Im Zusammenhang damit standen Begriffserklärungen wie die Frage der 'Realität' der im Minnesang auftretender Spielfiguren (Die frouwe der Minnesänger, 1980, Dörper oder Bauer, 1991) oder die Überprüfung etablierter germanistischer Namensformen wie 'Reinmar von Hagenau' oder 'Neidhart von Reuental' (War Reinmar 'von Hagenau' Hofsänger zu Wien? 1969, Neidhart: Nu ist vil gar zergangen ..., 1969) und daraus resultierend eine neue Sicht auf deren Stand und soziale Einordnung.

Im Fragekontext der Erfahrungswirklichkeit mittelalterlicher Sänger an den Höfen ihrer Gönner ergaben sich aus den Texten ablesbare Hinweise auf vielfältige Kommunikationsformen zwischen den Sängern und ihrem jeweiligen, immer wieder wechselnden Publikum (Der Stauferhof u. die mhd. Lyrik, 1978, Humor u. Ironie im Minnesang, 1982, Die Fehde zw. Walther u. Reinmar, 1986), v.a. aber Hinweise auf offensichtlich nach bestimmten Intentionen vorgenommene Textänderungen, die nicht einfach als Fehler der Überlieferung abgetan werden können, sondern die sich eher und logischer als Anpassung an ein bestimmtes Publikum oder aber als schöpferische Weiterentwicklung von Wortlaut, Thematik oder Form eines Textes erklären lassen, d.h. die Deutung von Doppel- und Mehrfachfassungen als vom Autor selbst geschaffene Liedvarianten. Diese Ergebnisse beruhen v.a. auf der genauen Untersuchung des gesamten aus dem 12. Jh. stammenden Textmaterials der Handschriften mit Hilfe eines Vergleichsschemas, wie es für einen Textkomplex S. 339ff. aufgeführt ist (Textkritik und Interpretation, 1964, Doppelfassungen bei 
Heinrich von Morungen, 1981). Weiter führte dies zur Überprüfung der sog. Unechtheitserklärungen, mit denen überlieferte Corpora beschnitten, auf éinen Ton und éine Stillage eingegrenzt wurden (Pseudo-Neidharte?, 1981) und zum Aufweis evtl. parodistisch abgewandelter Textübernahmen der Sänger untereinander (Stichwort 'Intertextualität': Eine Morungen-Parodie Walthers?, 1971, Steckt im Sumerlaten-Lied ..., 1968).

Zustimmung fanden mit der Zeit sowohl die These der in sich stimmigen Doppel- und Mehrfachfassungen, zumindest als Zeugnisse einer offenen, variablen Textgestaltung in der mittelalterlichen Vortragspraxis (vgl. v.a. H. HeInen, Mutabilität im Minnesang, 1989, und doppelte Textabdrucke in MF von MOSER/TervooREN) als auch die Widerlegung der Unechtheitserklärungen: Die mit den alten Forschungsaxiomen athetierten Lieder, insbes. die dem Schwank-Genre zugehörenden 'unechten' Texte können sich auch als lebensvolle Erweiterung eines mittelalterlichen Sänger-Repertoires erweisen, die, ad hoc eingesetzt, eine allzu 'hochgemute' Minnesang-Darbietung als eine Art Satyrspiel aufgelockert haben könnte (vgl. F. Maurer, Die 'Pseudoreimare', 1966, u.v.a. H. Tervooren, Reinmar-Studien, 1991, oder das Salzburger Neidhartprojekt).

Meine Kritik richtete sich also immer gegen die spekulative Schnellfertigkeit, mit der Texte, ohne daß nach ihrer philologisch-historischen Basis gefragt worden wäre, beurteilt oder für bestimmte Thesen vereinnahmt wurden. Notwendig erschien mir folglich auch eine Überprüfung der Editionstechnik, die auf zurechtkonjizierte Archetypen hinauslief. Die Texte sollten demgegenüber in ihrer historischen Offenheit, so, wie von den Handschriften angeboten, präsentiert werden. Ich habe in mehreren Ausgaben neue Wege gesucht, versucht, mit differenzierenden Apparaten und ausführlichen Kommentaren, z.T. mit Hilfe neuer, wie ich meine sachgerechterer Begriffe wie 'Stollenstrophe', 'Versgrenzenverschiebung' u.a., die mittelalterlichen Texte in ihrer Variabilität genauer als bisher zu beschreiben. Zwei der Arbeiten, welche diese Vorhaben begleiteten, sind hier ebenfalls aufgenommen, obwohl es inzwischen Gemeingut geworden ist, nach der historischen und überlieferungsgeschichtlichen Fundierung 
eines Textes zu fragen (Vom Edieren mhd. Lyrik, 1982, Zur Edition mhd. Lyrik, 1985).

Für die Reihenfolge der Aufsätze bot sich die Ordnung nach Erscheinungsjahren oder nach Sachbereichen an. Der mehr biographisch-chronologischen Reihung zog ich letztere vor, die m.E. das grundsätzliche methodische Vorgehen bei größeren Zusammenhängen über Gedichtinterpretationen bis hin zu Detailfragen deutlicher dokumentiert.

Beim Wiederlesen früherer Arbeiten fiel mir die gelegentliche Wiederholung bestimmter Sachverhalte, Argumente, Beobachungen, Funde oder Vergleiche auf, Resultat wohl der damals schwachen Resonanz meiner Thesen und meines ungebrochenen Überzeugungsdrangs. Dieser bedingte wohl auch ein gewisses Pathos in den frühen Arbeiten, dessen (für heutige Leser etwas befremdliche) Wirkung durch den damals üblichen pluralis majestatis noch unterstützt wird, eine Leseerfahrung, die ich mit den Herausgebern ähnlicher Sammlungen teile, die aber - nach Peter WAPNEWSKI - "als Reflex einer biographischen Position des Verfassers" nicht "in sprödere Valeurs umretuschiert" werden sollten. Ebenso verzichtete ich auch sonst auf Eingriffe. Meine heutigen Forschungspositionen sind in zwei Bändchen der Sammlung MeTzLeR, Minnesang (SM 244, 1989), Neidhart (SM 253, 1990), niedergelegt.

Schließlich ist es mir eine angenehme Pflicht, der Metzlerschen Verlagsbuchhandlung, vertreten durch Dr. Uwe Schweikert, für die Ermöglichung dieser Retrospektive zu danken. Ebenso danke ich Iris Guldan für die geduldige und fachmännische computergerechte Umsetzung der Manuskripte und die Anlage der Register, für weitere Mithilfe Sigrid Noelle und Isabell Marynik und wie stets meiner Frau. 